\title{
Comparison of Blind Source Separation Methods in Fast Somatosensory Evoked \\ Comparison of Blind Source Separation Methods in Fast Somatosensory Evoked
Potential Detection
}

3

4

.

.

3

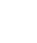
(1) (

${ }^{1}$ Department of Orthopaedics and Traumatology, The University of Hong Kong

${ }^{2}$ Department of Electrical and Electronic Engineering, The University of Hong Kong

Correspondence to: Dr. Y. Hu

12 Sandy Bay Road, Hong Kong

Duchess of Kent Children's Hospital

Department of Orthopaedics and Traumatology,

The University of Hong Kong

Pokfulam, Hong Kong

Tel: (852) 29740359

Fax: (852) 29740335

E-mail: yhud@hkusua.hku.hk

ACKNOWLEDGMENT: This work was supported in part by Hong Kong Research Grants Council (RGC) Competitive Earmarked Research Grants (GRF HKU 7130/06E) and The University of Hong Kong CRCG Funds. 5 . 7 
Abstract

2

Blind Source Separation (BSS) is a promising method for extracting somatosensory evoked

3 potential (SEP). Although various BSS algorithms are available for SEP extraction, few studies

4 have addressed the performance differences between them. In this study we compared the

5 performance of a number of typical BSS algorithms on SEP extraction from, via both computer

6 simulations and clinical experiment. The algorithms we compared included second-order blind

7 identification (SOBI), estimation of signal parameters via rotation invariance technique (ESPRIT),

8 algorithm for multiple unknown signals extraction (AMUSE), joint approximate diagonalization

9 of eigenmatrices (JADE), extended infomax, and fast independent component analysis (fastICA).

10 The performances of these BSS algorithms were determined by the correlation coefficients

11 between the true and the extracted SEP signals. There were significant differences in the

12 performances of the various BSS algorithms in a simulation study. In summary, SOBI using 6

13 covariance matrix denoting (SOBI6) was recommended as the most appropriate BSS method for

14 fast SEP extraction from noisy backgrounds.

15

16

17 Keywords: somatosensory evoked potentials, blind source separation, signal 18 processing 


\section{Introduction}

Somatosensory evoked potentials (SEPs) have been widely used for minimizing

3 possible risks during spinal surgery (Hu, et al., 2005, MacLennan and Lovely, 1995,

4 Nuwer, 1998). However, SEP signals recorded in the operating room are always

5 contaminated by noises of various sources, electrical and/or biological in nature

6 (Nuwer, 1998). This results in a low signal-to-noise ratio (SNR) of SEP (Lam, et al.,

7 2005) and makes real-time SEP extraction difficult, if not impossible. Filtering may

8 distort the SEP signals since it is overlapped with the noises in the frequency domain

9 (Komaromy, et al., 2002). The current standard way, ensemble averaging (EA),

10 increases SNR to a level at which the SEP signal is measurable (MacLennan and

11 Lovely, 1995, McKinley and Parker, 1991). However, depending on the quality of

12 recorded signals, EA method may require a large number (300-2000) of trials to

13 obtain an SEP interpretable waveform to clinicians and surgeons (American

14 Electroencephalographic Society, 1994). Moreover, for clinic use of SEP monitoring,

15 left and right sensory pathways are monitored respectively and a retesting (Strenge,

16 1989) is required to decrease false negative SEP monitoring. Thus, EA is

17 time-consuming, and consequently deficits may have already occurred before

18 acquiring enough trials (Deletis and Sala, 2008). In order to minimize the acquisition

19 time during surgery, new signal processing methods which provides a reliable SEP but

20 with fewer samples are needed (Ting, et al., 2006).

21 Many studies have been conducted for SEP extraction from noisy backgrounds

22 such as parametric modeling, adaptive filter, wavelet transform and blind source

23 separation (BSS). However a large number of records are still required in order to 
1 obtain an acceptable estimate in parametric modeling, whereas the performance of

2 adaptive filtering has been unsatisfactory, especially in a situation when recorded

3 signals are with a poor SNR (Liu, et al., 2007). Fortunately, improved performance

4 has been reported for BSS, which is a technique that recovers unknown source signals

5 from mixed and observed data sets (Iyer and Zouridakis, 2007), and BSS technique

6 has been suggested as a promising method for signal extraction from a noisy

7 background.

8 Since SEP responses are generally independent of neurophysiological artifacts and

9 background noises, BSS is able to extract SEP components from noisy measurements

10 (Lemm, et al., 2006). Furthermore, since BSS requires much fewer data trials than EA

11 (Ting, et al., 2006), it matches the purpose of fast extraction for SEP. Typical BSS

12 algorithms, including second-order blind identification (SOBI), estimation of signal 13 parameters via rotation invariance technique (ESPRIT), algorithm for multiple

14 unknown signals extraction (AMUSE), joint approximate diagonalization of 15 eigenmatrices (JADE), extended infomx, and fast independent component analysis 16 (fastICA), have been applied to other evoked potential extractions (Iyer and 17 Zouridakis, 2007, Lee, et al., 1999, Sutherland and Tang, 2006, Tang, et al., 2006,

18 Ting, et al., 2006) and may be applicable to SEP, even though it is weaker than other

19 evoked potentials. There are only a limited number of studies, however, comparing

20 the performances between the different BSS algorithms on the extraction of SEP.

21 Hence the aim of this study is to evaluate the performances of various BSS algorithms

22 on fast SEP extraction and to determine the most appropriate BSS algorithm(s) for 
11 practical applications.

2

3

4

2

10

11

12

13

14

15

16

17

18

19

20

21

22

23

24

25

26

27

28

29

30

31

32

33

34

35

36

37

38

39

40

41

42

43

44

45

46

47

48

49

50

51

52

53

54

55

56

57

58

59

60

61

62

63

64

65 


\section{Materials and Methods}

2

3

4

5

6

\subsection{SEP simulation}

\subsubsection{SEP template}

SEP signals were recorded from 15 scoliosis patient who underwent surgical correction. Ethical consents were obtained in advance. Following general anesthesia, nine needle electrodes were applied to the scalp at $\mathrm{Cz}, \mathrm{Fz}, \mathrm{C} 3, \mathrm{C} 4, \mathrm{P} 7, \mathrm{P} 8, \mathrm{O} 1, \mathrm{O} 2$ and Fz, prior to the commencement of spinal surgery. An additional pair of surface electrodes used as ground was applied to the skin over the cheek area. A total of eight

channels were recorded, with the reference to Fz. The stimulation for the recording of

SEPs was applied to the posterior tibial nerve, with a duration of $0.3 \mathrm{~ms}$, at a rate of 5.1 Hz, and a constant current of $10-30 \mathrm{~mA}$. The signal was amplified 100,000 times and band-pass filtered at 20-2000 Hz. All SEP signals were acquired and recorded by a computer with 12-bit resolution and $5 \mathrm{kHz}$ sampling rate. For each subject, the first $5 \mathrm{~ms}$ of data was removed before any processing because of stimulation artifact.

According to the suggestion of the American Electroencephalographic Society, (1994), the template signals for each subject were obtained by averaging 300 trials. Within the eight channel signals, Cz-Fz was the main channel for SEP monitoring, while the remaining seven channels provided extra information for BSS analysis. Therefore, the BSS performance mainly depends on the evaluation of the signal quality in the $\mathrm{Cz}-\mathrm{Fz}$ channel, in which the performances of the BSS methods are 
1 In this study, the background EEG and power-line noise were considered as two

2 main sources of noise, and were simulated and added to the SEP template (Chan, et al.,

3 1995). Then a simulated SEP signal was obtained for each subject. Three different

4 co-variations were used: 1) only EEG noise with $\mathrm{SNR}=-20 \mathrm{~dB}, 2$ ) only power-line

5 noise with $\mathrm{SNR}=-20 \mathrm{~dB}$, and 3) mixed (half-half) $\mathrm{EEG}$ and power-line noise with

$6 \quad \mathrm{SNR}=-20 \mathrm{~dB}$.

7 The EEG noise was simulated following an autoregressive model as follows (Qiu,

8 et al., 2006, Yu, et al., 1994):

9

$$
\begin{aligned}
\mathrm{EEG}_{i}(t) & =1.508 \mathrm{EEG}_{i}(t-1)-0.1587 \mathrm{EEG}_{i}(t-2) \\
& -0.3109 \mathrm{EEG}_{i}(t-3)-0.05 \text { 10EEG }_{i}(t-4)+n(t)
\end{aligned}
$$

where $\mathrm{EEG}_{i}(t)$ is the $i$-th channel of simulated EEG noise at the moment of $t$ and $n(\mathrm{t})$ is a random white Gaussian noise. The power-line noise was simulated as a $50 \mathrm{~Hz}$ sinusoid.

\subsection{Model and Methods of Blind Source Separation (BSS)}

The recorded multi-channel SEP measurements, denoted as $\mathbf{x}(t)=\left[\begin{array}{llll}x_{1}(t) & x_{2}(t) & \ldots & x_{M}(t)\end{array}\right]^{T}$, termed mixtures, were modeled as follows:

$$
\mathbf{x}(t)=\mathbf{A s}(t)+\mathbf{n}(t)
$$

where the entries of $\mathbf{s}(t)=\left[\begin{array}{llll}s_{1}(t) & s_{2}(t) & \ldots & s_{N}(t)\end{array}\right]^{T}$, i.e., the source signals were assumed independent of each other, $\quad \mathbf{n}(t)=\left[\begin{array}{lllll}n_{1}(t) & n_{2}(t) & \ldots & n_{M}(t)\end{array}\right]^{T}$ were independent additive white noise, and $\mathbf{A}$ is a $M \times N$ unknown full column-rank mixing matrix. The problem of estimating the unknown $\mathbf{A}$ and $\mathbf{s}(t)$ from the known measurement $\mathbf{x}(t)$ was termed blind source separation (BSS). In many approaches to BSS, A 
1 will be determined first, and then $\mathbf{s}(t)$ can be estimated as $\mathbf{A}^{+} \mathbf{x}(t)$. In order to

2 determine the mixing matrix $\mathbf{A}$, a number of methods including independent

3 component analysis (ICA) (Cardoso, 1998, Lemm, et al., 2006) and those based on

4 second-order statistics (SOS) (Belouchrani, et al., 1997, Joyce, et al., 2004, Tang, et

5 al., 2002, Ting, et al., 2006) can be applied.

6 ICA assumes that the sources are statistically independent and are non-Gaussian

7 (or at most one source signal is Gaussian). For non-trivial cases the sources can

8 theoretically be separated by ICA (Cardoso, 1998), which employs higher-order

9 statistics of the signals. If the sources are Gaussian, ICA will not work.

10 However, if the temporal structure of the source signals can be exploited, then

11 theoretically the sources can be separated by using second-order statistics (Tang, et al.,

12 2002, Ting, et al., 2006). Typical SOS-based blind source separation methods include

13 AMUSE, ESPRIT, and SOBI, where the source signals are assumed to be

14 uncorrelated but not necessarily non-Gaussian. The reason is that the resulting

15 algorithms are based on either generalized eigenvalue decomposition (EVD) or joint

16 diagonalization of two or more matrices constructed by second-order statistics of the

17 observed mixture signals.

18 The preprocessing of the data was performed before the application of BSS

19 methods. The first step is centering, i.e., removing the mean (time-average) of $\mathbf{x}(t)$

20 to make the mean zero. The centered measurements were then used for determining

21 the mixing matrix, and the original measurements were still used for source signal

22 estimation. The second step is the pre-whitening by singular value decomposition 
1 (SVD) of the measurement of data matrix $X=[x(1), x(2), \ldots, x(k)]$, i.e.,

2

21 we used $\mathrm{g}(\bullet)=\tanh (\bullet)$. respectively.

\subsubsection{FastICA} update rule

where $\mathbf{U}$ and $\Lambda$ are the left singular vector matrix and singular value matrix of $\mathbf{X}$

In the study of this paper, several typical blind source separation methods were employed. It is assumed that they were applied to the centered and pre-whitened signals without notification. That is to say, for ease of presentation, wherever we use $\mathbf{x}(t)$, we mean the pre-whitened $\tilde{\mathbf{x}}(t)$ defined by equation (3).

The fastICA algorithm (Hyvarinen, 1999, Oja and Yuan, 2006) maximizes the non-Gaussianity of estimates and uses nonlinear functions to efficiently estimate (approximate) negentropy, which is a non-negative function of the differential entropy. FastICA is based on a fixed-point iteration scheme to search for a maximum of the non-Gaussianity of the extracted signals $\hat{\mathbf{s}}(t)=\mathbf{W}^{T} \mathbf{x}(t)$. It can be summarized by the

$$
\mathbf{W}^{+}=\mathbf{E}\left\{\mathbf{x}(t) \mathbf{g}\left(\mathbf{W}^{T} \mathbf{x}(t)\right)\right\}-\mathbf{E}\left\{\mathbf{g}^{\prime}\left(\mathbf{W}^{T} \mathbf{x}(t)\right)\right\} \mathbf{W}
$$




\subsubsection{Joint approximate diagonalization of eigenmatrices}

The JADE algorithm (Cardoso and Souloumiac, 1993, Ziegaus and Lang, 2004) also assumes non-Gaussian independent source signals and utilizes joint diagonalization of a group of matrices constructed by fourth-order cumulants

$$
\begin{aligned}
\mathrm{Q} & =\operatorname{cum}\left(x_{i}, x_{j}, x_{k}, x_{l}\right) \\
& =\frac{\partial^{n} \Psi(x)}{\partial x_{i}^{n_{1}}, \partial x_{j}^{n_{2}}, \partial x_{k}^{n_{3}}, \partial x_{l}^{n_{4}}} \mid x_{i}=x_{j}=x_{k}=x_{l}=0
\end{aligned}
$$

which present additional information for higher order statistics, where $x_{\mathrm{i}}, x_{\mathrm{j}}, x_{\mathrm{k}}$, and $x_{1}$

are measurement data from arbitrary channels at arbitrary time points, $\Psi(x)$ is joint

8 probability distribution function, and $n=n_{1}+n_{2}+n_{3}+n_{4}$. The JADE algorithm aims to reduce mutual information contained in the cumulant matrices by looking for a rotation matrix, so that the cumulant matrices are as diagonal as possible. The joint diagonalization is determined by the Jacobi technique.

\subsubsection{Extended Infomax}

To extract the source signals from the mixtures by linear transformation, $\hat{s}(t)=\mathbf{W}^{T} \mathbf{x}(t)$, the extended infomax algorithm (Lee, et al., 1999) provides the following simple learning rule for the linear transformation matrix $\mathbf{W}$ with a fixed nonlinearity that can separate sources with a variety of distributions:

$$
\mathbf{W}^{+} \propto \begin{cases}{\left[\mathbf{I}-\tanh (\mathbf{u}) \mathbf{u}^{T}-\mathbf{u} \mathbf{u}^{T}\right] \mathbf{W}} & \text { :supergaussian } \\ {\left[\mathbf{I}+\tanh (\mathbf{u}) \mathbf{u}^{T}-\mathbf{u} \mathbf{u}^{T}\right] \mathbf{W}} & \text { :subergaussian }\end{cases}
$$


AMUSE (Tong et al., 1991) is based on the following representation of the auto-covariance matrix

$$
\mathbf{R}_{x}(\tau)=\mathbf{E}\left(\mathbf{x}(t+\tau) \mathbf{x}(t)^{T}\right)=\mathbf{A} \mathbf{E}\left(\mathbf{s}(t+\tau) \mathbf{s}(t)^{T}\right) \mathbf{A}^{T}=\mathbf{A} \mathbf{R}_{s}(\tau) \mathbf{A}^{T}
$$

4 A can then be determined by the eigenvalue decomposition of $\mathbf{R}_{x}(\tau)$.

\subsubsection{Second-order blind identification (SOBI)}

The SOBI algorithm (Belouchrani, et al., 1997, Joyce, et al., 2004, Tang, et al., 2005) is based on a set of time-lagged covariance matrices:

$$
\mathbf{R}_{x}(\tau)=\mathbf{E}\left(\mathbf{x}(t+\tau) \mathbf{x}(t)^{T}\right), \quad \tau \neq 0
$$

9 For independent sources, these matrices must be diagonal. To estimate the sources, a

10 joint diagonalization of the time-lagged covariance matrices is performed, similar to

11 the JADE algorithm. The approach of utilizing a set of $\tau$ values is adopted with the intention to avoid an inferior source separation, since there is no theoretically proven

13 choice of $\tau$ values. There is a flexibility in choosing the number of time-lagged 14 covariance matrices. In this study, we employed two combinations consistently. One method utilized $\mathbf{R}_{x}(1)$ and $\mathbf{R}_{x}(2)$, and was denoted as SOBI2. The other method utilized $\mathbf{R}_{x}(1), \quad \mathbf{R}_{x}(2), \quad \mathbf{R}_{x}(3), \quad \mathbf{R}_{x}(4), \quad \mathbf{R}_{x}(5), \quad \mathbf{R}_{x}(6)$, and was denoted as (ESPRIT)

Unlike the methods described above, ESPRIT (Chang, et al., 2000) acts directly on un-centered, original signals, so it is not affected by side effects brought on by 
1 pre-whitening. The JADE algorithm jointly diagonalizes a number of orthogonal

2 matrices, where the number can be larger than 2. The ESPRIT algorithm similarly

3 performs joint diagonalization; however, it jointly diagonalizes only two matrices that

4 are not necessarily orthogonal. The matrices, which ESPRIT aims to jointly

5 diagonalize, are covariance matrices of the original un-centered signals. The two

6 combinations consistently used in this paper were $\left\{\mathbf{R}_{x}(0), \mathbf{R}_{x}(1)\right\}$ and

$7\left\{\mathbf{R}_{x}(1), \mathbf{R}_{x}(2)\right\}$, and the corresponding ESPRIT methods are denoted as ESPRIT01

8 and ESPRIT12, respectively.

\subsection{Signal synthesis}

All the BSS algorithms described above were applied to extract SEP signals. The SEP-related components were selected then the SEP signal from channel Cz-Fz was extracted by inverse operation. The selection was performed by an experienced neurophysiologist with visual inspection, based on his clinical experiences. In an attempt to eliminate subjective bias, double-blind SEP components identification was also conducted in this study. Thus, all the SEP components were labeled and then mixed together and identified by the expert based on his experiences without knowing how to label them. For example, Figure 1 presents the ICA components, and No. 5 and No. 6 were identified as the SEP-related components (an SEP-liked waveform can be observed during 30-50ms).

The performances of different algorithms were compared in terms of the correlation coefficients between the known SEP templates and the estimated SEP signals (from $\mathrm{Cz}-\mathrm{Fz}$ ). The correlation coefficient ( $\mathrm{r}$ ) between $\mathbf{X}$ and $\mathbf{Y}$ is defined as 


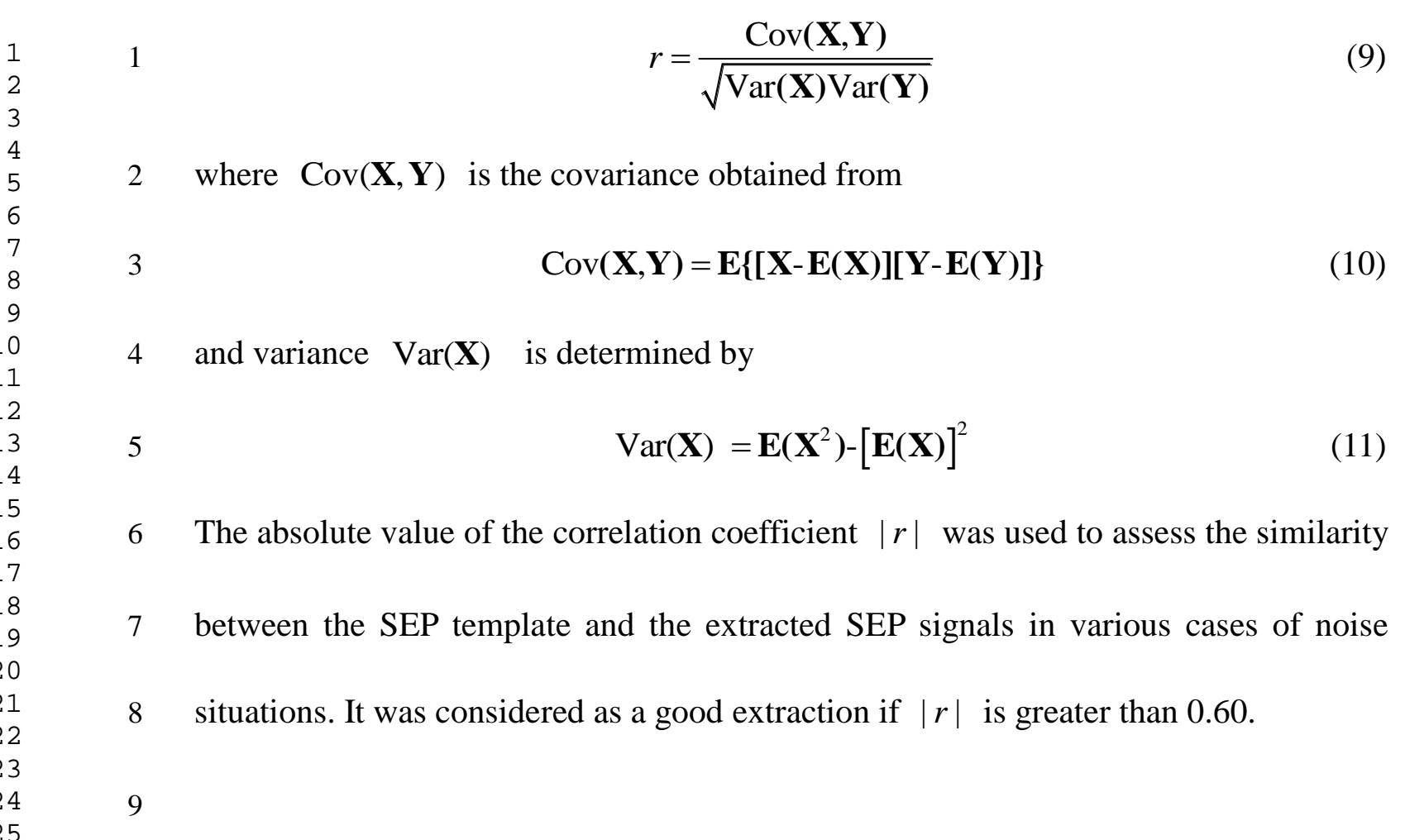




\section{Results}

\subsection{Simulation study}

The SEP templates were obtained from SEP recordings from 15 patients, and three different cases of noise were simulated according to the description in section 2.1.2. SEP signals were extracted from the simulated noisy SEP measurements by the BSS methods described in section 2.2 according to the procedure described in section 2.3. For example, using the data from one patient, the template SEP signal from $\mathrm{Cz}-\mathrm{Fz}$ determined after averaging 300 trials can be seen in Figure 2, the simulated SEP signal mixing with EEG and power-line noise with $\mathrm{SNR}=-20 \mathrm{~dB}$ can be seen in Figure $3(\mathrm{Cz}-\mathrm{Fz})$, the extracted SEP signal with SOBI6, similar to SOBI2, can be seen in Figure $4(\mathrm{Cz}-\mathrm{Fz})$, and the results of AMUSE can be seen in Figure $5(\mathrm{Cz}-\mathrm{Fz})$.

Just by looking at the graphs, it is clear that SOBI performed better than AMUSE. To quantify performance, the index of correlation coefficient described in section 2.3 was calculated for each combination of BSS algorithms and noise cases (Table 1). To compare the statistical differences of the correlation coefficients between these BSS methods, the one-way ANOVA was conducted and the results are shown in Table 2. When EEG was the only source of noise, the calculated correlation coefficients for SOBI2 $(0.67 \pm 0.07)$ and SOBI6 $(0.61 \pm 0.07)$ did not show significant differences between each other $(p>0.05)$, but they were significantly higher than that of other methods $(p<0.05)$. When the power-line noise was the only source of noise, the correlation coefficients were calculated $0.96 \pm 0.01$ in SOBI6, 0.95 \pm 0.02 in SOBI2, 0.93 \pm 0.08 in ESPRIT12, and 0.91 \pm 0.02 in ESPRIT01, without significant differences 
1 between each other, but they did show significant superiority to the correlation

2 coefficients in AMUSE, JADE and Extended infomax $(p<0.05)$. When the source of

3 noise was a combination of EEG and power-line, the correlation coefficients were

$4 \quad 0.77 \pm 0.03$ in SOBI6, which is significantly higher than that of any other method. The

5 statistical results suggest SOBI6 is the only outstanding method under this noise

6 situation. On the other hand, the fastICA algorithm seldom converged, thus the SEP

7 signal could not be extracted. For JADE and extended infomax, the correlation

8 coefficients were both less than 0.5 under all noise situations.

9 The success ratios, defined as the ratio of the number of cases with $|r|>0.60$ to

10 the total number of cases, of the different methods can be seen in Table 3 . When EEG

11 was the only source of noise, the success ratio of SOBI was greater than $50 \%$, when

12 only the power-line noise presented, the success ratio was $100 \%$ for ESPRIT and

13 SOBI. When there was a mixture of background EEG and power-line noise, SOBI6

14 was the only method that had a success ratio greater than $90 \%$, the success ratio of all

15 the other methods was less than 10\%. JADE and extended infomax showed extremely

16 unsatisfactory performances in terms of success ratios. This data suggests that SOBI6

17 is the most suitable method.

$18 \quad 3.2$ Experimental study

19 Experimental study was also conducted to examine the performance of various

20 BSS algorithms on real SEP data. To confirm the reliability of the extracted SEP by

21 the BSS algorithms, averaging was used. For each algorithm, the extracted SEP were averaged by $1,5,10,20$, and 40 trials. The averaged SEP by the BSS algorithms were 
1 presented in Fig. 6. In this figure, the single trial SEP is interpretable for SOBI6,

2 SOBI2, and ESPRIT01, while it is not for ESPRIT12, AMUSE, JADE and Infomax.

3 The latency was consistent along averaging for all algorithms except JADE, since the

4 SEP waveform by JADE cannot be identified. In Fig. 6, JADE and Infomax

5 performed unsatisfactory compared with other algorithms. They only extracted less

6 than 10 trials because of low successful ratio. For AMUSE, SOBI6, SOBI2,

7 ESPRIT01, and ESPRIT12, the SEP waveform (amplitude and latency) was stable

8 with more than 10 trials averaging. This indicated that the extracted SEP by SOS

9 algorithms is reliable.

10 All 15 subjects were employed in the experimental study. For each subject, all the

11 above mentioned BSS algorithms were used to extract SEP signals from 10

12 consecutive single trials. The correlation coefficient between the SEP template

13 (ensemble average of 300 trials) and the average of the 10 trials of BSS-extracted SEP

14 signals was computed in order to compare the performance of different BSS

15 algorithms. Table 4 shows the mean value, standard deviation and coefficient of

16 variation (CV) of the correlation coefficients. From Table 4, we can see that,

17 compared with other algorithms in terms of correlation coefficient, SOBI6 $(0.81 \pm 0.14)$

18 and SOBI2 $(0.72 \pm 0.21)$ present higher correlation with the SEP template, and

19 ESPRIT01 $(0.62 \pm 0.29)$ and ESPRIT12 $(0.61 \pm 0.28)$ are mildly acceptable. According

20 to the computed coefficient of variation, SOBI6 shows less variation than all the other

21 algorithms. Hence, SOBI6 is suggested to be used in SEP extraction. This result is

22 also consistent to that from the simulation study. Table 5 shows the mean value and 
1 standard deviation of correlation coefficient between two averaged 10 single trials

2 (the first one averaged 10 trials out of the first 50 trials, the second one averaged 10

3 trials out of the last 50 trials). In this table, SOBI showed the highest reproducibility

4 of the extracted SEP, which is $0.94 \pm 0.03$ for SOBI6 and is $0.88 \pm 0.07$ for SOBI2.

5 AMUSE and ESPRIT performed similar. JADE and Informax presented the lowest

6 reproducibility.

7 


\section{Discussion}

The aim of this study is to determine the most appropriate BSS algorithm(s) for obtaining a good SEP signal from a small number of trials of SEP recordings, and to determine which BSS method provides a sufficient high quality waveform comparable to the conventional ensemble averaging. In simulation study, the performance of seven different BSS algorithms under three different noise situations were assessed in terms of correlation coefficients between SEP templates and the extracted SEP signals. When background EEG was the only source of noise, SOBI presented acceptable results, while the performances of all the other methods were unsatisfactory. When only power-line noise was present, all methods except for JADE and infomax showed a promising performance, the performances of SOBI and ESPRIT were extremely good. Finally, when there was a mixture of EEG and power-line noise, the performance of SOBI6 was outstanding. On the other hand, the performances of JADE, infomax, and fastICA were not as good under the all noise situations.

The experimental results also presented that the single trail extracted SEPs by SOBI are more satisfactory than those by other algorithms. In Table 4, SOBI showed the highest correlation with the SEP averaged by 300 trials. In Table 5, SOBI performed the highest reproducibility between two SEPs which were averaged by 10 extracted single trials. These results indicate that SOBI is not only performed better than other BSS algorithms but also presented a satisfactory reproducibility in SEP extraction. It suggests that SOBI could be the method of choice, whereas JADE, 
1 infomax, and fastICA are not recommended.

2

3

4

5 other fields (Hyvarinen, 1999, Lee, et al., 1999), their performances in SEP extraction

6 are unsatisfactory. This is probably the case because, according to our experience, in

7 order to get a reliable estimate of HOS, thousands of data points are needed, while

8 only hundreds of data points are required for a reliable estimate of second order 9 statistics (SOS). In this study, the latency of SEP is under 100 milliseconds, and when

Although higher-order statistic (HOS)-based algorithms such as fastICA, extended infomax and JADE are robust algorithms and have been successfully used in many the sampling time is $100 \mathrm{~ms}$ with a sampling rate of $5 \mathrm{kHz}$ and the length of the sweeps was only 500 points, that is not enough for a reliable estimate of HOS but it is sufficient for SOS estimation. It should be noted that the problem of data shortage cannot be solved completely by merely improving the sampling frequency. The increase of sampling frequency will introduce increased dependency among data points, and compared to independent data, such dependency will require more data points to estimate the statistics with the same accuracy. This goes to show that additional information cannot be introduced by simply improving the sampling frequency. It has been previously established that for signals with limited data points, SOS-based BSS algorithms may perform better than HOS-based algorithms (chunqi Chang, 2000), This explains why AMUSE, SOBI, and ESPRIT, all methods based on SOS, perform much better than HOS-based algorithms. Similar conclusions have also been reported in several other studies (Ting, et al., 2006), (Zavala-Fernandez, et al., 
1 2006). The results of the present study indicate that the performance of SOBI is

2 superior to that of JADE and this is partly consistent to those of previous reports

3 ((Zavala-Fernandez, et al., 2006)) which stated JADE was marginally less successful

4 but SOBI and fastICA presented similar performance. In this report, however, the data

5 point was 62500 , much longer than the data point of the present study, which was only

6500 , therefore it is sufficient for a reliable estimate of HOS. Moreover, in our

7 simulation study, even though the background noise is Gaussian, the source signals

8 (SEP template) are non-Gaussian. Gaussian noise is actually better for HOS-based

9 methods, suggesting that HOS algorithms such as JADE, Extended infomax and

10 FastICA are not suitable for SEP extraction. It must also be noted that SOS-based BSS

11 algorithms have their own limitations, as they require the source signals to have

12 colored spectra, and the correlation and shift correlation matrices to have distinct

13 eigenvalues. Fortunately, these requirements seem to be satisfied in the application of

14 SEP extraction.

From the comparison of SOS-based BSS algorithms SOBI, AMUSE, and ESPRIT (chunqi Chang, 2000), SOBI2 and SOBI6 seems to be superior to other methods when the noises are uncorrelated. SOBI has also been successfully applied to neurological signals extraction from background noises (Joyce, et al., 2004, Sutherland and Tang, 2006, Tang, et al., 2006). Among these SOS-based blind source separation compared methods, each has its own characteristics, and they are different on how they utilize the correlation matrices which are second order statistics. AMUSE is the most basic one, which use the zero-lag correlation matrix and another correlation matrix of 
1 nonzero lag. The ESPRIT method uses two correlation matrices both of nonzero lags

2 so as to cope with color noise more efficiently. Different from AMUSE and ESPRIT,

3 the family of SOBI methods use more than two correlation matrices. Though the

4 computation complexity will be increased if we work on more correlation matrices,

5 the additional information brought by additional correlation matrices will improve the

6 anti-noise robustness of the blind source separation. That's why SOBI algorithms are

7 more suitable than AMUSE and ESPRIT for our application of SEP signal processing.

8 However, one may not want to use too many correlation matrices, because not only

9 the increased computation complexity but also the possible decrease of performance

10 on accuracy since correlation matrices at large time lags may be dominated by noise

11 and thus will deteriorate the performance of the algorithm. Therefore, in practice we

12 need to choose an appropriate number of correlation matrices for the SOBI algorithm,

13 and through our experimental study it is found that SOBI2 and SOBI6, which use 2

14 and 6 correlation matrices, respectively, are good choices for SEP signal processing.

15 In fact, practical users of SOBI can use any number of correlation matrices from 2 to

16 6. The choice is just a trade-off between computational complexity and performance.

17 In this study, SOBI, when applied specifically to short-time SEP signals, demonstrated

18 outstanding performance compared with all other BSS algorithms, providing

19 additional support to the findings of previous studies. Further studies investigating

20 automatic SEP-related component recognition are required in order to avoid 21 subjective bias.

22 From the clinical point of view, the reproducibility is also concerned. It is of 
1 importance to evaluate the reliability of the clinical use of SEP. Because it associates

2 with the borders for changes of latency and amplitude for giving relevant warning

3 signals with high sensitivity and specificity to avoid false-negative and -positive

4 warnings (Strahm, et al., 2003). Luk et al. (2001) also stated that a reliable monitoring

5 technique should be reproducible. From our experimental results, SOBI6 presented

6 the highest reproducibility of $0.94 \pm 0.03$ in terms of correlation coefficient between

7 two SEPs (averaged by 10 single trials), compared with other algorithms. It indicates

8 that SEPs extracted by SOBI6 are more reproducible than by other algorithms.

9 Hence, based on both the simulation and experimental results, SOBI6 is the

10 suggested method for SEP fast extraction based on simulation and experimental

11 studies. However, it should be noted that real-time SEP extraction in its true sense is

12 online and automatic. Therefore, it is suggested that there should be a further study

13 which focuses more on the automatic SEP extraction based on SOBI. 


\section{Conclusion}

2 In this study, we applied blind source separation (BSS) methods to the extraction

3 of somatosensory evoked potentials (SEP) from multi-channel noisy measurements. A

4 number of different BSS methods were studied and their performances were

5 compared under three different noise situations: EEG noise only, power-line noise

6 only, and a combination of EEG and power-line noise. Results of simulation study

7 demonstrated that HOS-based BSS algorithms, infomax, JADE, and fastICA, are not

8 robust to the three noise simulations, ESPRIT01 is only robust to power-line noise,

9 and SOBI2 is not robust to the mixed EEG and power-line noise. On the other hand,

10 the SOBI6 algorithm demonstrated outstanding performance under all three noise

11 situations. Experimental study also showed that the performance of SOBI6 was

12 indeed superior to all the other algorithms. In addition, SOBI presented the highest

13 reproducibility among BSS algorithms. From both studies, SOBI6 was recommended

14 as the most appropriate BSS method for fast SEP extraction from noisy backgrounds. 


\section{References}

2 American Electroencephalographic Society. Guideline eleven: guidelines for intraoperative monitoring of sensory evoked potentials. J Clin Neurophysiol. 1994; 11:77-87.

Belouchrani A, Abed-Meraim K, Cardoso JF and Moulines E. A blind source separation technique using second-order statistics. IEEE Trans Sig Process. 1997; 45:434-444.

Cardoso JF. Blind signal separation: statistical principles. Proceedings of the IEEE. 1998; 86:2009-2025.

Cardoso JF and Souloumiac A. Blind beamforming for non-Gaussian signals. Radar and Signal Processing, IEE Proceedings F. 1993; 140:362-370.

Chan FH, Lam FK, Poon PW and Qiu W. Detection of brainstem auditory evoked potential by adaptive filtering. Med Biol Eng Comput. 1995; 33:69-75.

Chang CQ, Ding Z, Sze FY, and Chan HY. A matrix-pencil approach to blind separation fo colored nonstationary signals. IEEE Trans on Signal Processing. 2000; 48(3):900-907.

Deletis V and Sala F. Intraoperative neurophysiological monitoring of the spinal cord during spinal cord and spine surgery: a review focus on the corticospinal tracts. Clin Neurophysiol. 2008; 119:248-264.

Hu Y, Lam BS, Chang CQ, Chan FH, Lu WW and Luk KD. Adaptive signal enhancement of somatosensory evoked potential for spinal cord compression detection: an experimental study. Comput Biol Med. 2005; 35:814-828.

Hyvarinen A. Fast and robust fixed-point algorithms for independent component analysis. Neural Networks, IEEE Transactions on. 1999; 10:626-634.

Iyer D and Zouridakis G. Single-trial evoked potential estimation: Comparison between independent component analysis and wavelet denoising. Clin Neurophysiol. 2007; 118:495-504.

Joyce CA, Gorodnitsky IF and Kutas M. Automatic removal of eye movement and blink artifacts from EEG data using blind component separation. Psychophysiology. 2004; 41:313-325.

Komaromy AM, Brooks DE, Dawson WW, Kallberg ME, Ollivier FJ and Ofri R. Technical issues in electrodiagnostic recording. Vet Ophthalmol. 2002; 5:85-91.

Lam BS, Hu Y, Lu WW, Luk KD, Chang CQ, Qiu W and Chan FH. Multi-adaptive filtering technique for surface somatosensory evoked potentials processing. Med Eng Phys. 2005; 27:257-266.

Lee TW, Girolami M and Sejnowski TJ. Independent component analysis using an extended infomax algorithm for mixed subgaussian and supergaussian sources. Neural Comput. 1999; 11:417-441.

Lemm S, Curio G, Hlushchuk Y and Muller KR. Enhancing the signal-to-noise ratio of ICA-based extracted ERPs. IEEE Trans Biomed Eng. 2006; 53:601-607.

Liu H, Hu Y, Chang CQ and Luk KDK. Fast extraction of Somatosensory Evoked Potential based on Second Order Blind Identification. In: Engineering in Medicine and Biology Society, 2007. EMBS 2007. 29th Annual International Conference of the IEEE, 2007. 5457-5460.

Luk KD, Hu Y, Wong YW and Cheung KM. Evaluation of various evoked potential techniques for spinal cord monitoring during scoliosis surgery. Spine. 2001; 26:1772-1777.

MacLennan AR and Lovely DF. Reduction of evoked potential measurement time by a TMS320 based adaptive matched filter. Med Eng Phys. 1995; 17:248-256.

McKinley CA and Parker PA. A beamformer for the acquisition of evoked potentials. IEEE Trans Biomed Eng. 1991; 38:379-382.

Nuwer MR. Spinal cord monitoring with somatosensory techniques. J Clin Neurophysiol. 1998; 15:183-193. 
Oja E and Yuan Z. The fastICA algorithm revisited: convergence analysis. IEEE Trans Neural Netw. 2006; 17:1370-1381.

Qiu W, Chang C, Liu W, Poon PW, Hu Y, Lam FK, Hamernik RP, Wei G and Chan FH. Real-time data-reusing adaptive learning of a radial basis function network for tracking evoked potentials. IEEE Trans Biomed Eng. 2006; 53:226-237. Strahm C, Min K, Boos N, Ruetsch Y and Curt A. Reliability of perioperative SSEP recordings in spine surgery. Spinal Cord. 2003; 41:483-489.

Strenge H. The repeat reliability of somatosensory evoked potentials. EEG EMG Z Elektroenzephalogr Elektromyogr Verwandte Geb. 1989; 20:147-152.

Sutherland MT and Tang AC. Reliable detection of bilateral activation in human primary somatosensory cortex by unilateral median nerve stimulation. Neuroimage. 2006; 33:1042-1054.

Tang A, Sutherland M and Wang Y. Contrasting single-trial ERPs between experimental manipulations: improving differentiability by blind source separation. Neuroimage. 2006; 29:335-346.

4 Tang AC, Liu JY and Sutherland MT. Recovery of correlated neuronal sources from EEG: the good and bad ways of using SOBI. Neuroimage. 2005; 28:507-519. Magnetoencephalography: Single-Trial Response Onset Times. NeuroImage. 2002; 17:1773-1789.

Ting KH, Fung PC, Chang CQ and Chan FH. Automatic correction of artifact from single-trial event-related potentials by blind source separation using second order statistics only. Med Eng Phys. 2006; 28:780-794.

$\mathrm{Yu} \mathrm{XH}, \mathrm{He} \mathrm{ZY}$ and Zhang YS. Time-varying adaptive filters for evoked potential estimation. IEEE Trans Biomed Eng. 1994; 41:1062-1071.

Zavala-Fernandez H, Sander TH, Burghoff M, Orglmeister R and Trahms L. Comparison of ICA algorithms for the isolation of biological artifacts in magnetoencephalography. In, 2006 Charleston, SC, United States. 511-518.

Ziegaus $\mathrm{C}$ and Lang EW. A neural implementation of the JADE algorithm (nJADE) using higher-order neurons. Neurocomputing. 2004; 56:79-100. 
Figure legends

2

Fig. 1 Independent components of SOBI

4

5 Fig. 2 SEP template

6

7

Fig. 3 Simulated SEP signal

8

9 Fig. 4 SOBI6 extracted SEP

10

11 Fig. 5 AMUSE-extracted SEP

12

13 Fig. 6 Single trial SEP signal and 5,10,20 and 40 trials averaging after different BSS

14 extraction methods

15

20

21 


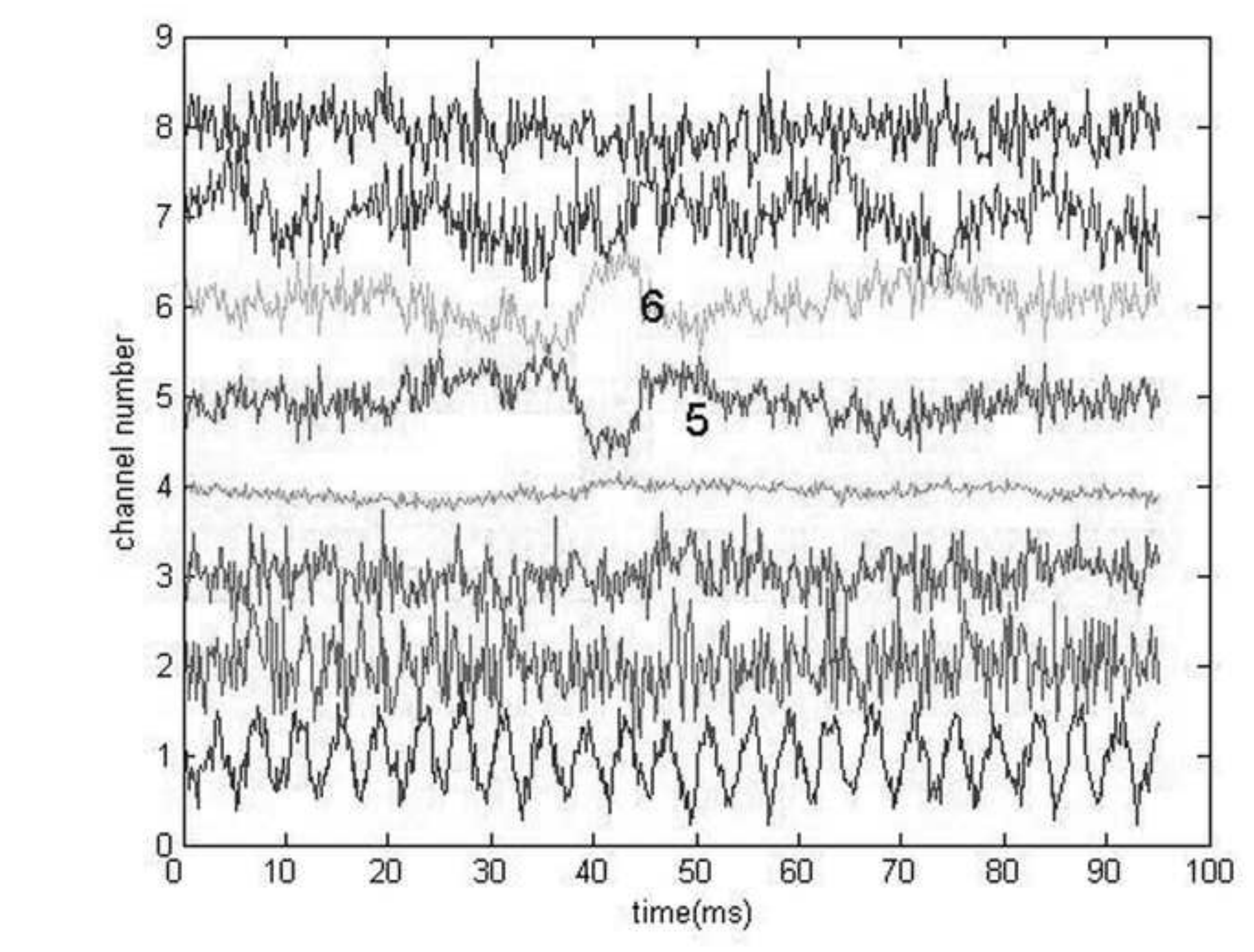

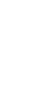




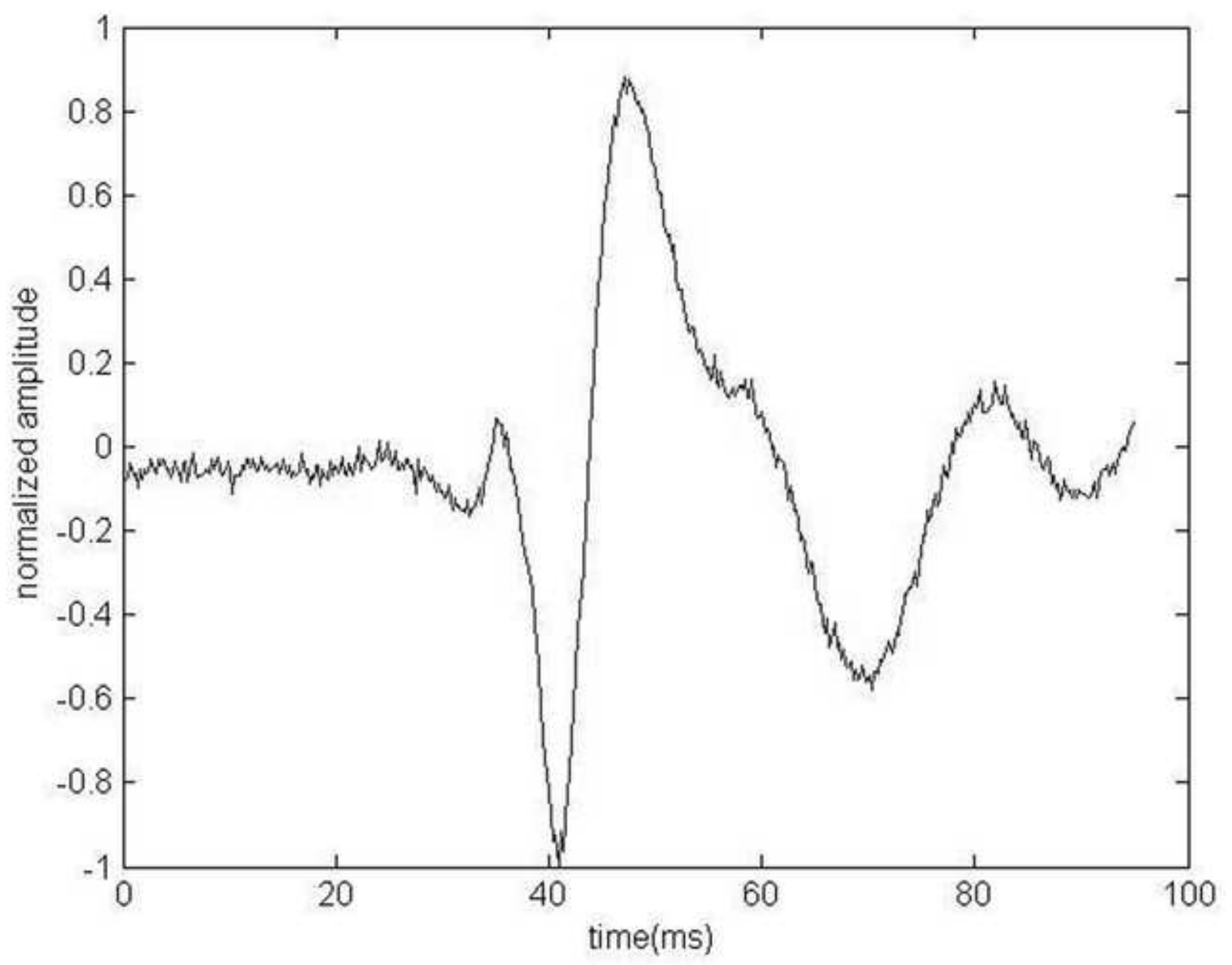




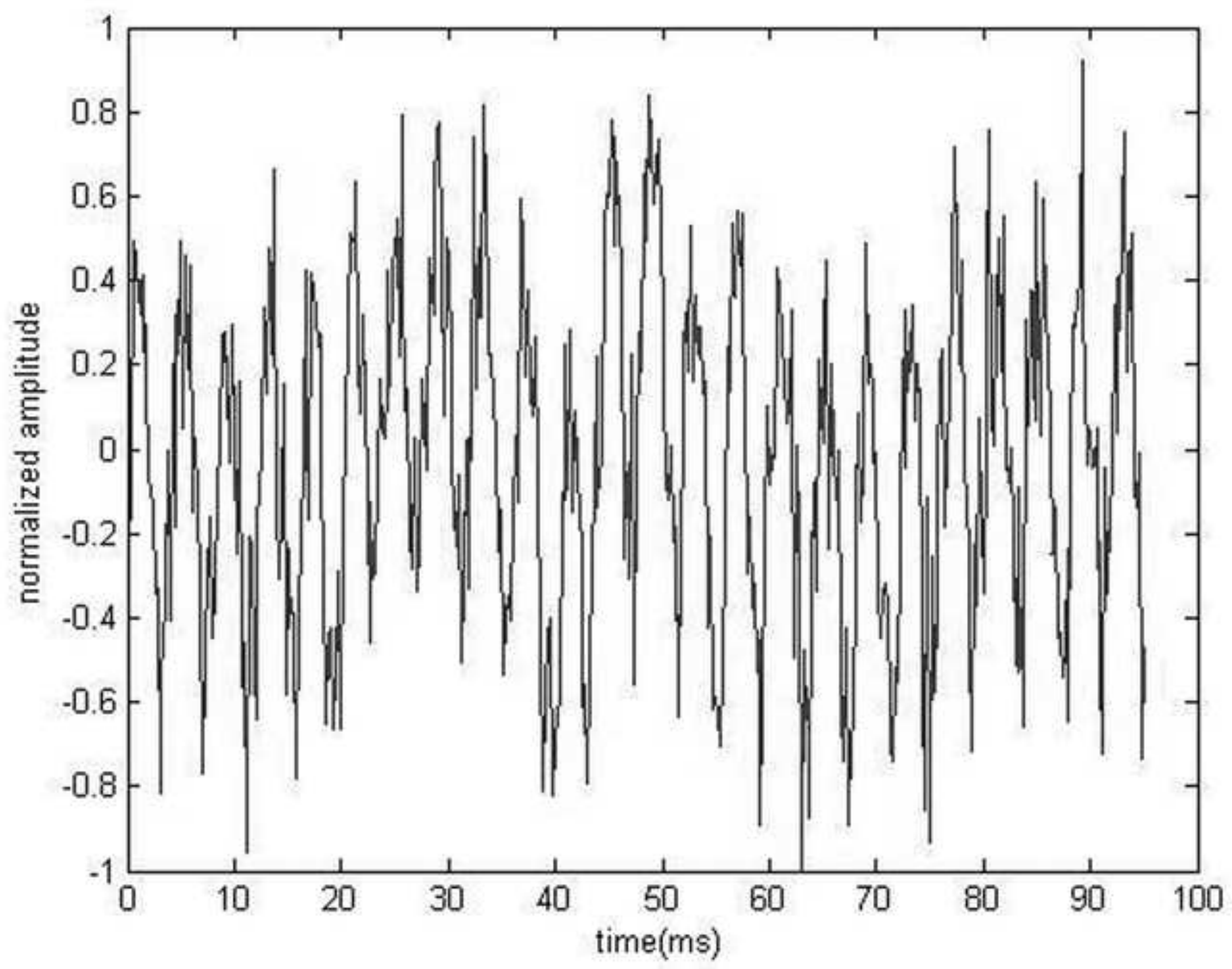




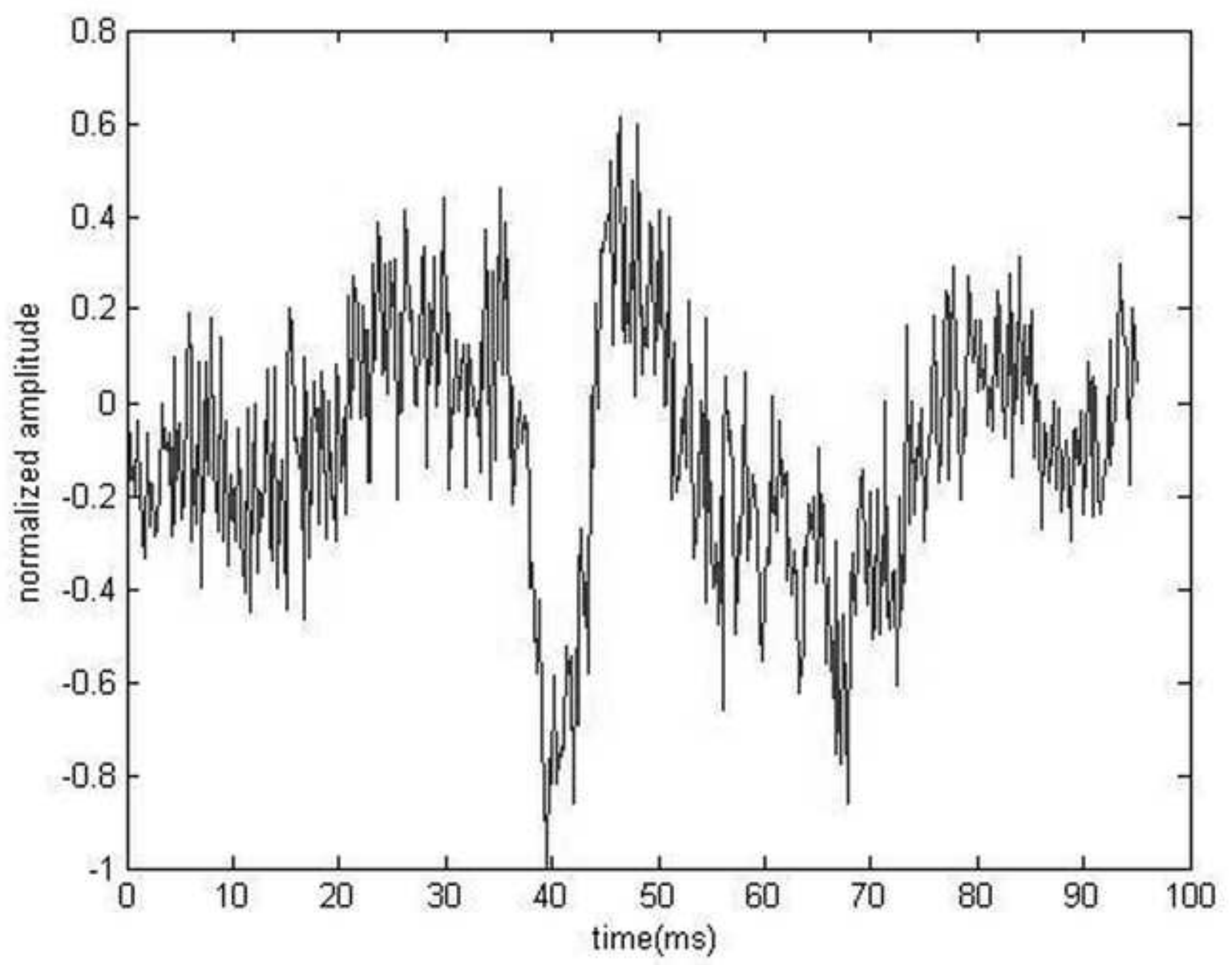




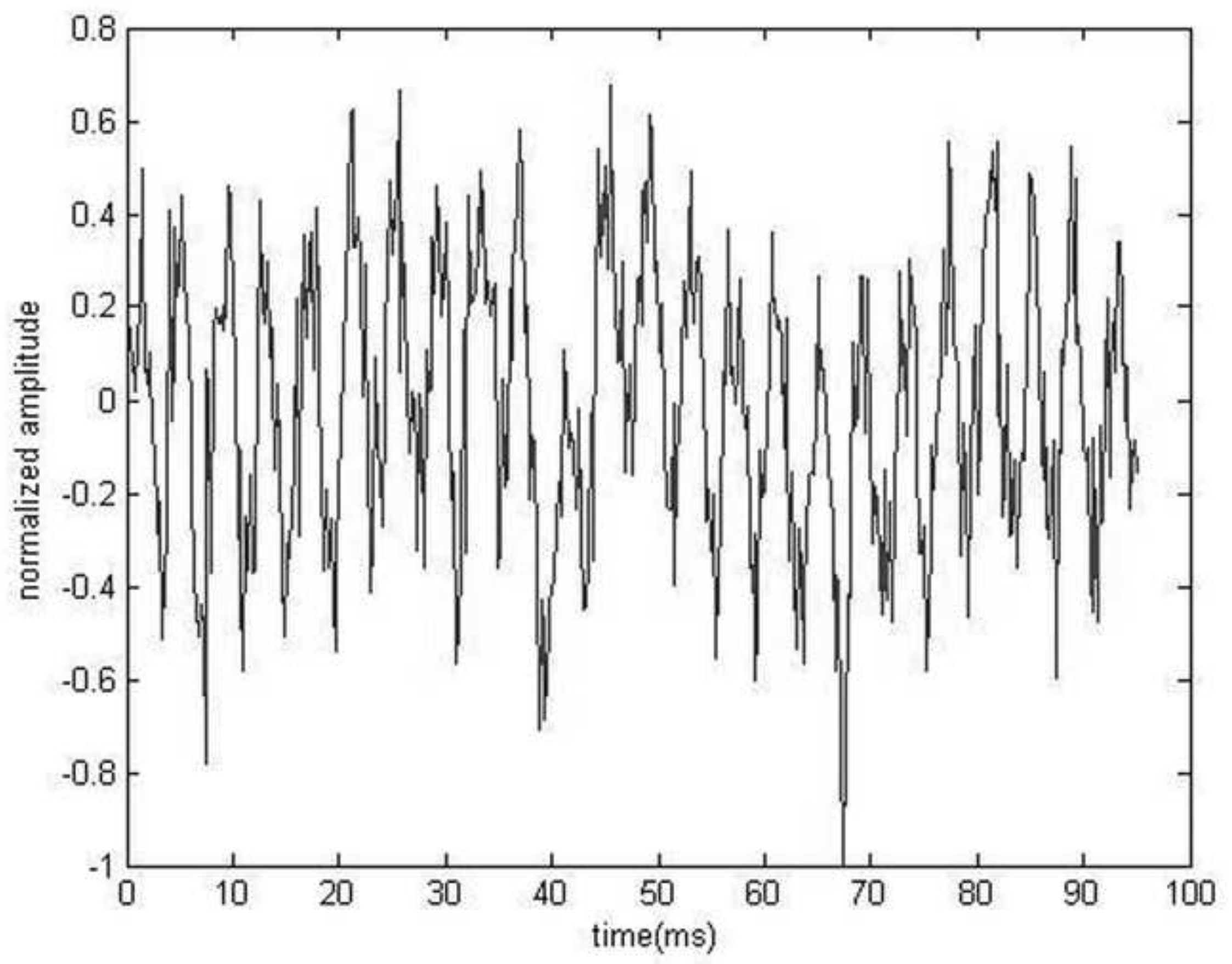


Figure6

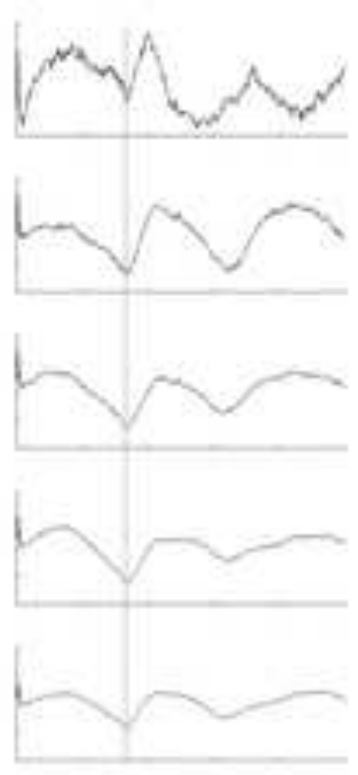

SOBI6

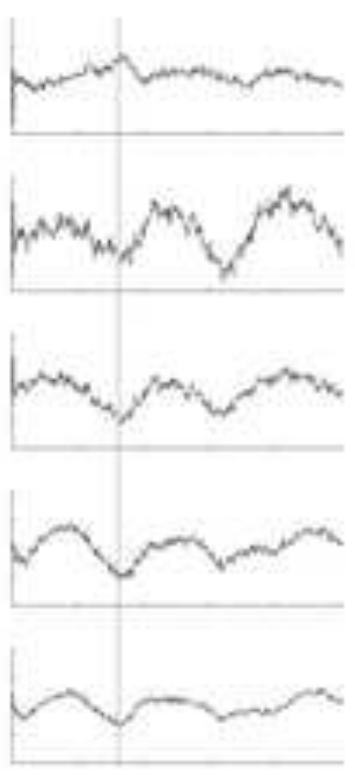

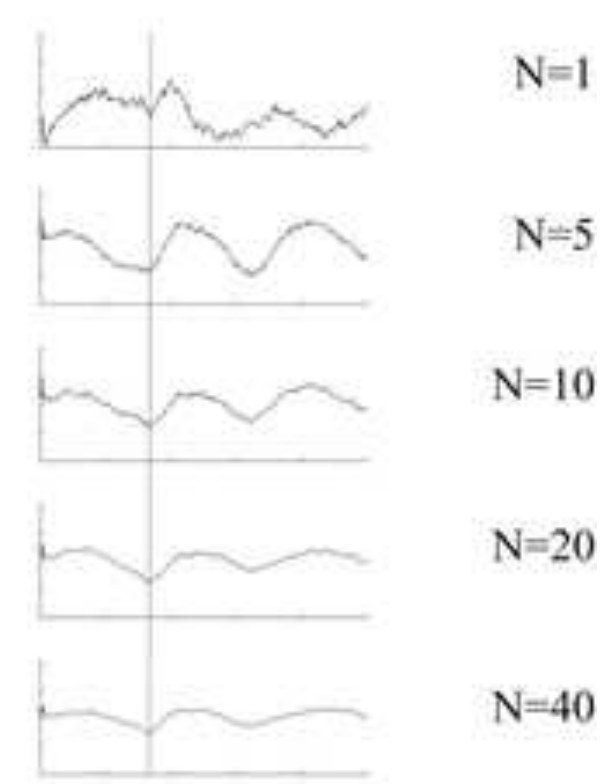

ESPRIT01

Infomax

\section{JADE}

ESPRIT12

Infomax

TADE

SOBI2

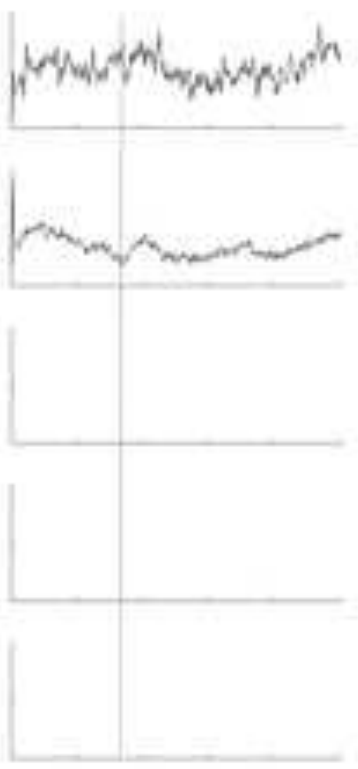

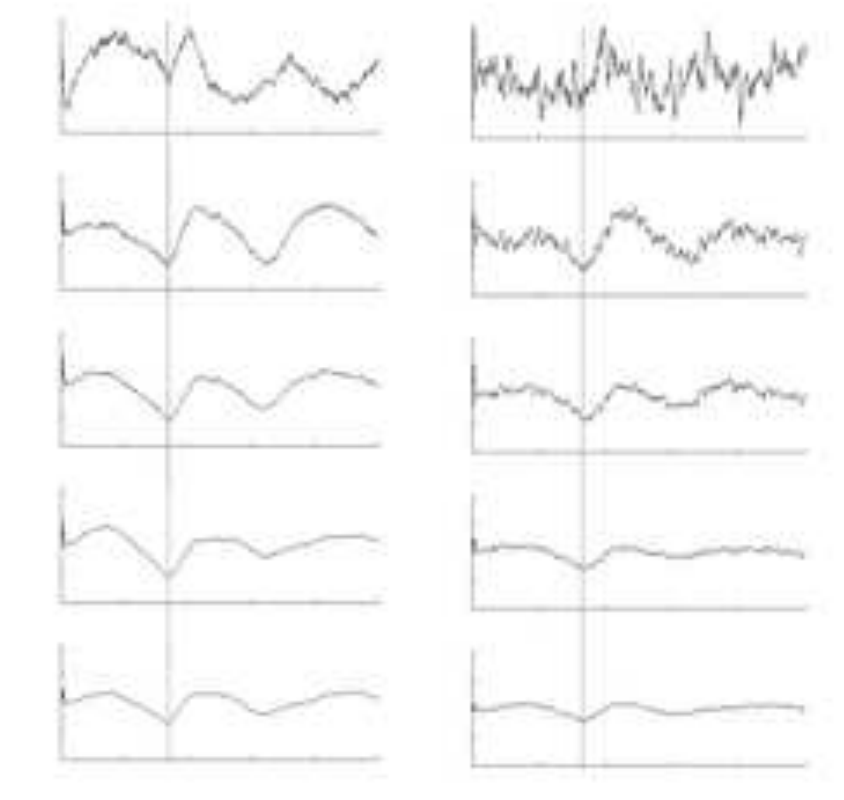

AMUSE

JADE
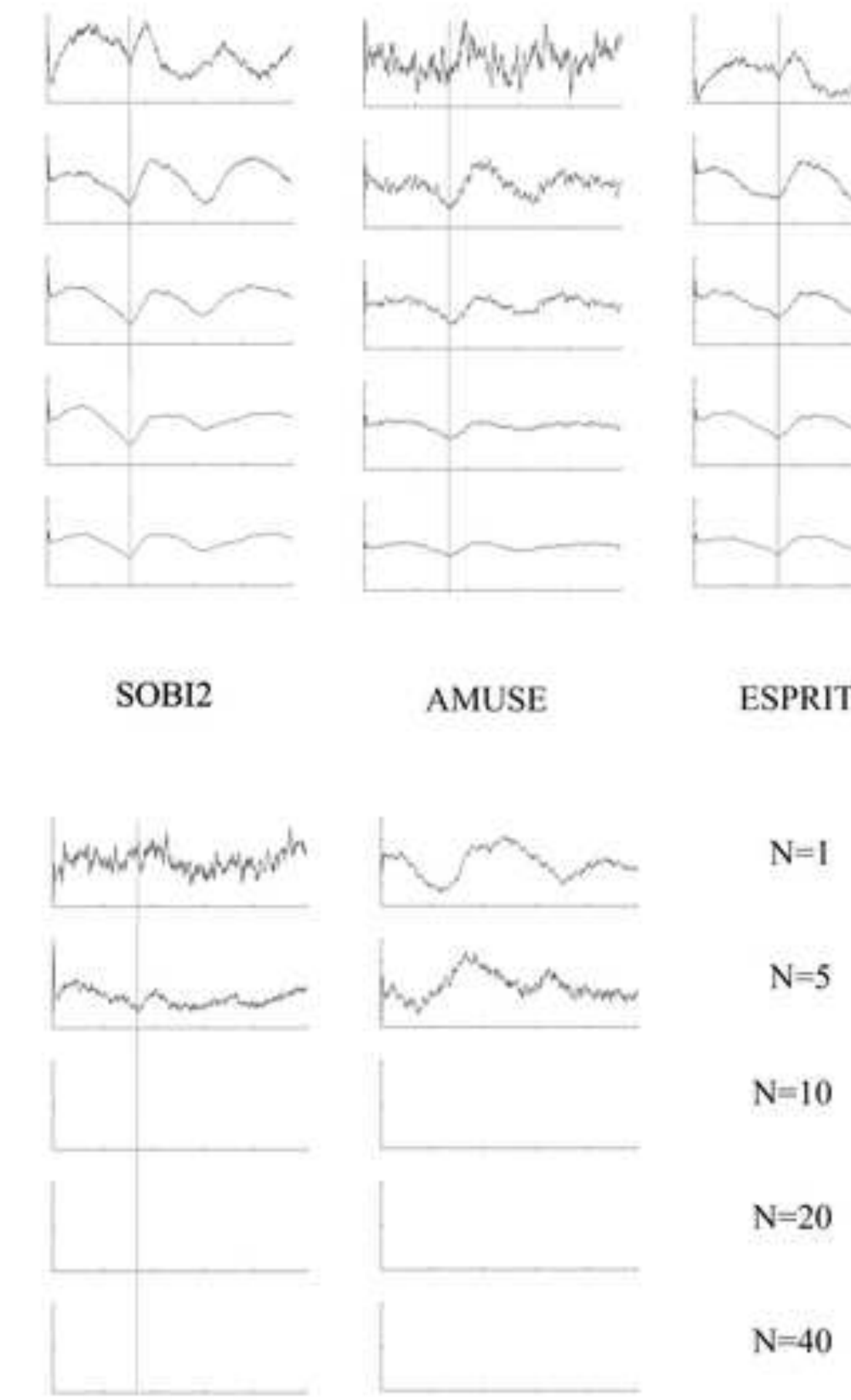

\section{AMUSE}
$\mathrm{N}=1$
$\mathrm{V}=5$
$N=10$
$\mathrm{N}=20$
$=40$

.


Table 1: Correlation coefficients between template and BSS-extracted SEP under different noise conditions

\begin{tabular}{cccccccc}
\hline Noise & AMUSE & ESPRIT01 & ESPRIT12 & SOBI2 & SOBI6 & JADE & Extended Infomax \\
\hline EEG & $0.16 \pm 0.24$ & $0.56 \pm 0.12$ & $0.27 \pm 0.26$ & $0.67 \pm 0.07$ & $0.61 \pm 0.07$ & $0.37 \pm 0.04$ & $0.15 \pm 0.13$ \\
$50 \mathrm{~Hz}$ & $0.53 \pm 0.27$ & $0.91 \pm 0.04$ & $0.93 \pm 0.08$ & $0.95 \pm 0.02$ & $0.96 \pm 0.01$ & $0.37 \pm 0.07$ & $0.46 \pm 0.28$ \\
EEG $+50 \mathrm{~Hz}$ & $0.24 \pm 0.16$ & $0.21 \pm 0.35$ & $0.32 \pm 0.26$ & $0.36 \pm 0.26$ & $0.77 \pm 0.03$ & $0.36 \pm 0.08$ & $0.25 \pm 0.09$ \\
\hline
\end{tabular}


Table 2: Statistical significance ( $p$ values) between methods

\begin{tabular}{|c|c|c|c|c|c|c|}
\hline & ESPRIT01 & ESPRIT12 & SOBI2 & SOBI6 & JADE & Extended Infomax \\
\hline \multicolumn{7}{|c|}{ EEG } \\
\hline AMUSE & $<0.001 *$ & NS & $<0.001 *$ & $<0.001 *$ & $0.04 *$ & NS \\
\hline ESPRIT01 & & $<0.001 *$ & NS & NS & $<0.001 *$ & $<0.001 *$ \\
\hline ESPRIT12 & & & $<0.001 *$ & $<0.001 *$ & NS & NS \\
\hline SOBI2 & & & & NS & $<0.001 *$ & $<0.001 *$ \\
\hline SOBI6 & & & & & $<0.001 *$ & $<0.001 *$ \\
\hline JADE & & & & & & $0.04 *$ \\
\hline \multicolumn{7}{|c|}{$50 \mathrm{~Hz}$} \\
\hline AMUSE & $<0.001 *$ & $<0.001 *$ & $<0.001 *$ & $<0.001 *$ & NS & NS \\
\hline ESPRIT01 & & NS & NS & NS & $<0.001 *$ & $<0.001 *$ \\
\hline ESPRIT12 & & & NS & NS & $<0.001 *$ & $<0.001 *$ \\
\hline SOBI2 & & & & NS & $<0.001 *$ & $<0.001 *$ \\
\hline SOBI6 & & & & & $<0.001 *$ & $<0.001 *$ \\
\hline JADE & & & & & & NS \\
\hline \multicolumn{7}{|c|}{$\mathrm{EEG}+50 \mathrm{~Hz}$} \\
\hline AMUSE & NS & NS & NS & $<0.001 *$ & NS & NS \\
\hline ESPRIT01 & & NS & NS & $<0.001 *$ & NS & NS \\
\hline ESPRIT12 & & & NS & $<0.001 *$ & NS & NS \\
\hline SOBI2 & & & & $<0.001 *$ & NS & NS \\
\hline SOBI6 & & & & & $<0.001 *$ & $<0.001 *$ \\
\hline JADE & & & & & & NS \\
\hline
\end{tabular}

*Statistical significance at $p<0.05$. Insignificant results are marked NS. 
Table 3: Success ratio of SEP extraction under different noise conditions

\begin{tabular}{cccccccc}
\hline Noise/SNR(dB) & AMUSE(\%) & ESPRIT01(\%) & ESPRIT12(\%) & SOBI2(\%) & SOBI6(\%) & JADE(\%) & $\begin{array}{c}\text { Extended } \\
\text { Infomax }(\%)\end{array}$ \\
\hline EEG/-20 & 0 & 40 & 0 & 60 & 53 & 0 & 0 \\
50Hz/-20 & 47 & 100 & 100 & 100 & 100 & 0 & 33 \\
EEG+50Hz/-20 & 0 & 0 & 0 & 6.7 & 93 & 0 & 6.7 \\
Overall & 15.7 & 46.7 & 33.3 & 55.7 & 82 & 0 & 13 \\
\hline
\end{tabular}


Table 4: correlation coefficients between SEP template and averaged extracted SEP signals for all algorithms

\begin{tabular}{cccccccc}
\hline & AMUSE & ESPRIT01 & ESPRIT12 & SOBI2 & SOBI6 & JADE & Extended Infomax \\
\hline Mean & 0.5693 & 0.6236 & 0.6052 & 0.7241 & 0.8066 & 0.3696 & 0.4309 \\
std & 0.2557 & 0.2856 & 0.2819 & 0.2094 & 0.1448 & 0.2442 & 0.2388 \\
CV & $45 \%$ & $46 \%$ & $47 \%$ & $29 \%$ & $18 \%$ & $66 \%$ & $55 \%$ \\
\hline
\end{tabular}


Table 5: Correlation coefficient between two averaged extracted SEP signals for all subjects

\begin{tabular}{cccccccc}
\hline & AMUSE & ESPRIT01 & ESPRIT12 & SOBI2 & SOBI6 & JADE & $\begin{array}{c}\text { Extended } \\
\text { Infomax }\end{array}$ \\
\hline mean & 0.62 & 0.62 & 0.68 & 0.88 & 0.94 & $0.26^{*}$ & $0.36^{*}$ \\
std & 0.24 & 0.27 & 0.16 & 0.07 & 0.03 & $0.17^{*}$ & $0.22^{*}$ \\
CV & $39 \%$ & $44 \%$ & $24 \%$ & $8.0 \%$ & $3.2 \%$ & $65 \%^{*}$ & $61 \%^{*}$ \\
\hline
\end{tabular}

* denotes that the mean and standard deviation are not calculated for all subjects, since some subjects cannot successfully extract more than 10 trails. 